\title{
Application of Grey Comprehensive Entropy Weight Clustering Method in Performance Evaluation of Banks
}

\author{
Jie Yuyang ${ }^{1, a^{*}, \text { Dong Fenyi }}{ }^{2, b}$ \\ ${ }^{1}$ College of Information and Management Science, Henan Agricultural University, Zhengzhou, \\ China \\ ${ }^{2}$ College of Information and Management Science, Henan Agricultural University, Zhengzhou, \\ China \\ a18237129839@163.com, bdfenyi@163.com
}

Keywords: Entropy weight, Grey clustering method, Management performance evaluation.

\begin{abstract}
The performance of commercial banks not only affects their own development, but also relates to the operation of the national macro economy. Through the analysis of the banking business. From the four aspects of profitability, management ability, growth and development ability and the security of bank assets, establish evaluation index system. With reference to the information entropy, the weight of each index is determined from two aspects: subjective and objective, and the evaluation method of grey cluster evaluation is used to evaluate the performance of the 16 listed banks. The results show that the overall performance of listed banks in China is in a good level.
\end{abstract}

\section{灰色综合熵权聚类法在银行经营绩效评价中的应用 \\ 介宇扬 ${ }^{1, a^{*}}$,董奋义 $2, b$, \\ 1河南农业大学信息与管理科学学院, 郑州, 河南, 中国 \\ 2中国河南农业大学信息与管理科学学院, 郑州, 河南, 中国 \\ a18237129839@163.com, bdfenyi@163.com}

关键词：熵权; 灰色聚类方法; 经营绩效评价

中文摘要. 商业银行的经营绩效不仅影响自身的发展也关系到国家宏观经济的运行。通过对 银行经营业务的特点进行分析，从盈利能力、管理能力、成长发展能力以及银行资产的安全 性四个方面选取指标建立评价指标体系。借鉴信息熵的思想，从主观和客观两个方面综合确 定各指标的权重, 运用灰色聚类评估法对16家上市银行的经营绩效进行综合评价。结果显示 我国上市银行经营绩效整体处于较好的水平。

\section{1. 引言}

随着中国经济体制改革的不断深入，金融市场的竞争也愈演愈烈。商业银行作为国民经 济的支柱, 对国家经济发展和社会稳定具有重要的作用。已上市的商业银行相对其它未上市 的银行，在经营模式、治理结构以及信息公开等方面更具有规范性，因此对银行类上市公司 进行绩效评价，可以了解整个银行业的经营情况，从而为金融业的快速发展提供参考。

综合已有的研究发现, 多数文献在评价指标的选取上, 参照的是一般企业经营绩效的评 价标准, 忽略了银行的行业特殊性。在方法的选择方面, 有些方法可能会出现量化结果和定 性分析结果不一致的现象，有些方法的计算过程和计算量过于复杂。对此本文基于 16 家上市 
银行 2015 年最新的年报数据, 在构建商业银行经营绩效评价体系时综合考虑银行的业务特点, 构建出科学合理的商业银行经营绩效评价指标体系。同时本文借鉴信息熵的思想, 提出一种 将主观分析与客观分析相结合构建指标综合权重的方法, 该方法是一种较为全面的赋权方法, 并将综合熵权法与灰色定权聚类相结合，克服了灰色聚类方法中指标权重过于主观的问题。

\section{2. 银行类上市公司经营绩效评价指标体系构建和数据收集}

科学的评价指标体系不仅可以公正地反映上市银行的经营绩效，还可以为广大投资者和 利益相关者提供决策有用的信息, 因此建立科学合理的评价指标体系, 选取适当的评价方法, 对评价商业银行的经营绩效具有十分重要的意义。

影响商业银行经营绩效的因素较多, 目前尚未形成一个统一完整的评价指标体系。在构 建银行类上市公司经营绩效评价指标体系时, 应结合银行的经营特点, 并遵循科学性、全面 性、可比性和数据可获得性。高鹏 [1]在对我国商业银行经营绩效评价中选用主营业务利润率、 资本收益率、净资产收益率等指标。田金金, 隋杨 $[2]$ 则选用资产收益率、费用利润率、流动性 比率等指标对银行经营绩效进行综合评价。

常见的银行经营绩效评价指标体系多以会计利润为中心，且在指标的选择上多采用普通 企业的经营绩效评价指标。虽然会计利润对评价银行经营绩效十分重要, 但片面强调会计利 润在一定程度上存在局限性, 忽略了银行的行业特点以及银行成长发展的能力。本文综合考 虑银行的盈利性、流动性、安全性和成长发展能力, 在六个常用绩效评价指标: 资产利润率、 资产流动性比例、资本充足率、成本收入比、总资产增长率和资本积累率的基础上，增加了 可以反映银行业务特点的非利息收入占比和不良贷款率两个指标。由于成本收入比和不良贷 款率两个指标属于逆向指标, 在允许的范围内越小越好, 因此为了便于统计分析, 在进行建 模前对这两个指标进行处理, 分别用收入成本比和非不良贷款率来代替, 具体数据见表 1 。

表1 2015年我国16家上市银行经营绩效评价指标

\begin{tabular}{|c|c|c|c|c|c|c|c|c|}
\hline \multirow[b]{2}{*}{ 银行 } & \multicolumn{8}{|c|}{ 指标 } \\
\hline & $\begin{array}{l}\text { 资产利润 } \\
\text { 率 }(\mathrm{x} 1)\end{array}$ & $\begin{array}{c}\text { 非利息收 } \\
\text { 入占比 } \\
(\mathrm{x} 2)\end{array}$ & $\begin{array}{l}\text { 收入成本 } \\
\text { 比 }(x 3)\end{array}$ & $\begin{array}{l}\text { 流动性比 } \\
\text { 率 }(\mathrm{x} 4)\end{array}$ & $\begin{array}{l}\text { 资本充足 } \\
\text { 率 }(x 5)\end{array}$ & $\begin{array}{l}\text { 非不良贷 } \\
\text { 款率（x6） }\end{array}$ & $\begin{array}{l}\text { 总资产增 } \\
\text { 长率 }(x 7)\end{array}$ & $\begin{array}{l}\text { 资本积累 } \\
\text { 率 }(x 8)\end{array}$ \\
\hline 民生银行 & 1.07 & 35.74 & 68.78 & 44.72 & 11.49 & 98.40 & 12.59 & 25.43 \\
\hline 宁波银行 & 1.03 & 19. 98 & 65.97 & 42.44 & 13.29 & 99.08 & 29.30 & 32.00 \\
\hline 中信银行 & 0.90 & 28.04 & 72.15 & 44.97 & 11.87 & 98.57 & 23.76 & 19.58 \\
\hline 招商银行 & 1.14 & 32.13 & 72.33 & 65.67 & 11.91 & 98.32 & 15.71 & 14.76 \\
\hline 浦发银行 & 1.10 & 22.89 & 78.14 & 33.50 & 12.23 & 98.44 & 20.22 & 21.14 \\
\hline 兴业银行 & 1.04 & 22.36 & 78.41 & 56.80 & 11.19 & 98.54 & 20.25 & 21.60 \\
\hline 工商银行 & 1.30 & 27.20 & 74.51 & 35.05 & 15.22 & 98.50 & 7.76 & 16.89 \\
\hline 农业银行 & 1.07 & 18.66 & 66.72 & 44.50 & 13.40 & 97.61 & 11.40 & 17.36 \\
\hline 平安银行 & 0.93 & 31.26 & 68.69 & 54.29 & 10.94 & 98.55 & 14.67 & 23.33 \\
\hline 交通银行 & 0.99 & 25.62 & 69.64 & 42.90 & 13.49 & 98.49 & 14.15 & 13.55 \\
\hline 建设银行 & 1.30 & 24.36 & 73.02 & 44.17 & 15.39 & 98.42 & 9.59 & 15.44 \\
\hline 南京银行 & 1.02 & 17.53 & 75.90 & 55.44 & 13.11 & 99.17 & 40.46 & 59.88 \\
\hline 光大银行 & 1.00 & 28.66 & 73.09 & 54.90 & 11.87 & 98.39 & 15.74 & 24.83 \\
\hline 中国银行 & 1.12 & 30.65 & 71.70 & 48.60 & 14.06 & 98.57 & 10.26 & 14.72 \\
\hline 华夏银行 & 0.98 & 21.69 & 64.99 & 39.14 & 10.85 & 98.48 & 9.13 & 15.95 \\
\hline 北京银行 & 1.00 & 18.82 & 75.01 & 34.76 & 12.27 & 98.88 & 21.02 & 21.53 \\
\hline
\end{tabular}




\section{3. 银行类上市公司经营绩效评价方法及模型}

\section{1 基于信息摘的综合权重确定}

熵的概念源自于热力学，用来描述离子或分子运动的不可逆现象。C.E.Shannon将熵引入 信息论，信息熵用以反映信息无序化的程度，度量数据所提供的有效信息。摘权法是一种客 观赋权方法，根据各指标的变异程度，利用信息熵计算各指标的熵权。当评价对象在某项指 标上的值相差较大, 熵值较小, 说明该指标提供的有效信息量较大, 该指标的权重应较大; 反 之若评价对象在某项指标上的值相差较小, 熵值较大, 说明该指标提供的信息量较少, 该指标 的权重也应较小 [3]。

设有 $\mathrm{m}$ 个评价对象, $\mathrm{n}$ 个评价指标，原始评价对象的指标值可以用矩阵 $X=\left(x_{i j}\right)_{m \times n}$ 来表示。 由于评价指标体系中各指标在量纲等方面可能存在较大的差异, 同时指标可能存在正向指标 和逆向指标的区别, 因此在计算之前, 应先对不同类型的指标分别进行标准化处理如:

$$
\begin{aligned}
& R_{i j^{*}}=\frac{x_{i j^{*}}-\min \left(x_{i j^{*}}\right)}{\max \left(x_{i j^{*}}\right)-\min \left(x_{i j^{*}}\right)} \\
& R_{i j^{*}}=\frac{\max \left(x_{i j^{*}}\right)-x_{i j^{*}}}{\max \left(x_{i j^{*}}\right)-\min \left(x_{i j^{*}}\right)}
\end{aligned}
$$

其中 $j^{*}$ 为定值且 $j^{*} \in 1,2, \ldots, n$ 。标准化后的指标值用矩阵 $R_{i j}=\left(r_{i j}\right)_{m \times n}$ 表示。利用熵权法计算指标 权重的步骤为:

步骤1：计算第 $\mathrm{j}$ 指标下第 $\mathrm{i}$ 个对象的指标值的比重 $p_{i j}, p_{i j}=r_{i j} / \sum_{i=1}^{m} r_{i j}$;

步骤 2 : 计算 $\mathrm{j}$ 指标的熵值 $e_{j}, e_{j}=-k \sum_{i=1}^{m} p_{i j} \cdot \ln p_{i j}$, 其中 $k=\frac{1}{\ln m}$;

步骤3: 计算 $\mathrm{j}$ 指标的熵权 $w_{j}, w_{j}=\left(1-e_{i j}\right) / \sum_{j=1}^{n}\left(1-e_{i j}\right)$;

步骤4: 结合定性分析确定的主观权重 $\eta_{j}$, 计算指标的综合权重 $\beta_{j}=\frac{\eta_{j} \cdot w_{j}}{\sum_{j=1}^{n} \eta_{j} \cdot w_{j}}$ 。

\section{2 基于综合熵权的灰色聚类法}

灰色聚类评估法是灰色系统理论中最早发展并得以广泛应用的一门技术，一直是灰色系 统理论中讨论较多的灰色技术之一。灰色聚类评估方法是由邓聚龙在 1987 年基于灰数的白化 函数理论创立的。张岐山 [4] 最早将信息熵引入灰色系统中, 提出了灰关联熵的概念。随后很 多学者对灰关联熵进行了完善和发展, 将其广泛应于用交通、军事、灾害预防等领域。米传 民、刘思峰、党耀国等 [5]将信息熵与灰色聚类相结合, 提出了灰色熵权聚类决策方法。刘力、 周建中、杨莉等 [6]将其运用于洪灾评估中。王生龙、邹志红 [7] 将其应用于水质综合评价中。 毕和政、陆键、朱胜雪 [8]提出了组合赋权的灰色聚类法, 并应用在一级公路平交口安全评价 中。钱丽丽、刘思峰、谢乃明 [9]提出了基于熵权和区间灰数信息的灰色聚类模型。

一般灰色定权聚类法定义有 $\mathrm{m}$ 个聚类对象， $\mathrm{n}$ 个聚类指标， $\mathrm{s}$ 个不同灰类，聚类对象 $\mathrm{i}(\mathrm{i}=1, \ldots, \mathrm{m})$ 关于指标 $\mathrm{j}(\mathrm{j}=1, \ldots, \mathrm{n})$ 的观测值为 $x_{i j}$, 将对象 $\mathrm{i}$ 归为第 $\mathrm{k}(\mathrm{k}=1,2, \ldots, \mathrm{s})$ 灰类, 称为灰色聚 类。灰色定权聚类的计算步骤为 $[10]$ :

步骤1: 确定 $\mathrm{j}$ 指标 $\mathrm{k}$ 子类的白化权函数 $f_{j}^{k}().(j=1,2, \ldots, n ; k=1,2 \ldots, s)$;

步骤 2 : 确定各指标的聚类权 $\eta_{j}(j=1,2, \ldots, n)$;

步骤3: 由前两步得到的白化权函数, 聚类权以及对象 $\mathrm{i}$ 关于指标 $\mathrm{j}$ 的观测值 $x_{i j}$, 计算灰色定权聚类系数

$$
\sigma_{i}^{k}=\sum_{j=1}^{n} f_{j}^{k}\left(x_{i j}\right) \cdot \eta_{j}
$$


步骤4: 若 $\max _{1 \leq k \leq s}\left\{\sigma_{i}^{k}\right\}=\sigma_{i}^{k^{*}}$, 则断定对象 $i$ 属于灰类 $k^{*}$ 。

当聚类指标的意义、量纲不同且在数量上差异悬殊时, 采用灰色定权聚类法可以得到较 好的聚类结果，但是该方法在权重的处理方面过于主观。已有研究虽然将信息熵与灰色聚类 相结合，但仅从数据本身所含带的信息中确定权重，没有将熵权法中客观分析和现实中的主 观情况相结合。因此本文借鉴信息熵的思想，在灰色定权聚类法的基础上，提出一种将主观 分析和客观分析相结合确定权重的灰色综合熵权聚类法, 即在专家给予主观权重 $\eta_{j}$ 后, 运用 综合熵权法计算得到综合权重 $\beta_{j}$ ，然后再进行一般灰色定权聚类法中的后两步。

\section{3 银行类上市公司经营绩效评价模型}

本文将16家上市银行的经营绩效水平划分为低等水平、中低水平、中高水平和高等水平 四个类别, 分别用下限测度白化权函数、适中测度白化权函数（包括中低和中高水平）、上 限测度白化权函数来测度。将评价对象、指标和灰类进行编号, 给出对象 $\mathrm{i}(\mathrm{i}=1,2,3 \ldots . .16)$ 的 $\mathrm{j}$ $(\mathrm{j}=1,2, \ldots, 8)$ 指标 $\mathrm{k}(\mathrm{k}=1,2,3,4)$ 灰类的白化权函数。

通过德尔菲法确定八个指标的主观权重 $\eta_{j}$ 分别为: $0.25 、 0.07 、 0.08 、 0.20 、 0.11 、 0.13$ 、 $0.08 、 0.08$ 。其次运用MATLAB软件计算得到八个指标的熵权 $w_{j}$ 为：0.0411、0.1583、0.1871、 $0.0741 、 0.1924 、 0.0964 、 0.1132 、 0.1374$ 。然后利用公式 $(6)$ 计算得到最终的综合权重 $\beta_{j}$ 为: $0.10 、 0.11 、 0.14 、 0.14 、 0.20 、 0.12 、 0.09 、 0.10$ 。最后运用灰色聚类软件得到我国16家上市 银行经营绩效水平的灰色聚类系数和聚类结果, 如表2所示。

表2我国16家上市银行经营绩效水平的灰色聚类结果

\begin{tabular}{|c|c|c|c|c|c|}
\hline \multirow{2}{*}{ 银行 } & \multicolumn{4}{|c|}{ 聚类系数 } & \multirow{2}{*}{ 聚类结果 } \\
\cline { 1 - 4 } & 第1类 & 第2类 & 第3类 & 第4类 & \\
\hline 宁波银行 & 0.29 & 0.31 & 0.21 & 0.21 & 2 \\
\hline 中信银行 & 0.25 & 0.16 & 0.13 & 0.31 & 4 \\
\hline 招商银行 & 0.10 & 0.23 & 0.35 & 0.21 & 3 \\
\hline 浦发银行 & 0.22 & 0.17 & 0.15 & 0.35 & 4 \\
\hline 兴业银行 & 0.20 & 0.34 & 0.12 & 0.24 & 2 \\
\hline 工商银行 & 0.29 & 0.10 & 0.14 & 0.29 & 3 \\
\hline 农业银行 & 0.48 & 0.20 & 0.26 & 0.01 & 4 \\
\hline 平安银行 & 0.30 & 0.14 & 0.17 & 0.27 & 1 \\
\hline 交通银行 & 0.15 & 0.30 & 0.19 & 0.03 & 2 \\
\hline 建设银行 & 0.22 & 0.23 & 0.22 & 0.30 & 4 \\
\hline 南京银行 & 0.11 & 0.10 & 0.13 & 0.59 & 4 \\
\hline 光大银行 & 0.12 & 0.20 & 0.21 & 0.24 & 4 \\
\hline 中国银行 & 0.19 & 0.00 & 0.21 & 0.50 & 4 \\
\hline 华夏银行 & 0.77 & 0.14 & 0.02 & 0.00 & 1 \\
\hline 北京银行 & 0.25 & 0.20 & 0.13 & 0.27 & 4 \\
\hline
\end{tabular}

\section{4. 结束语}

16 家上市银行中属于高等发展水平灰类的有 8 家，分别是宁波银行、招商银行、工商银行、 建设银行、南京银行、光大银行、中国银行和北京银行, 说明我国上市银行经营绩效大部分 处于较好水平。其中中国银行、工商银行及建设银行属于我国五大国有股份制银行, 在资产 
规模, 政策待遇以及管理能力方面都具有领先优势。其余五家银行虽属于地区性股份制银行, 但在资产安全、盈利收入以及经营管理等方面的能力较好，大部分指标也均处于较高水平。

属于中高水平灰类的有中信银行和兴业银行。兴业银行费用管理能力以及资产的流动性 都比较强, 该行的成本收入比控制的最好, 资产流动比率居16家银行中的第二, 但该行资本 充足率较差, 其余指标均处于中等偏上水平。中信银行大部分指标处于中等偏上水平, 其中 总资产增长率较高, 但是资本利润率过低, 说明该行利润收入的增长与资产的增长不匹配, 因此中信银行在扩大规模的同时应注意同时提高自身的利润收入。

属于中低水平灰类的有民生银行、浦发银行和交通银行。民生银行的非利息业务开展的 较好, 该行非利息收入占比最高, 但是其他各项指标处于中等偏下水平。浦发银行各方面的 管理不够均衡, 各项指标所处水平参差不齐落差较大, 导致该行在总体的绩效评价中处于中 低水平。交通银行除资本充足率还可以外, 其余各项指标均处于中等偏下水平, 其中资本积 累率最低, 说明交行资本积累能力较差。

属于低等灰类水平的银行有农业银行、平安银行和华夏银行。该类别的银行大部分指标 均处于较差水平。农业银行的不良贷款率最高, 应格外注意风险管理, 提高贷款质量, 同时 应加大不良贷款的拔备覆盖率做好应对风险的准备。平安银行和华夏银行值得注意的是资本 充足率, 均已接近监管的红线水平。银行大规模集中放贷使资本充足率出现较大程度的下滑, 为了防范由于信贷问题引发新的金融风险，银行必须进一步提升现有的资本金充足率。

\section{致谢}

本文为国家自然科学基金一般项目(71571157)的阶段性成果之一。

\section{References}

[1]Gao Peng, Performance evaluation of listed commercial banks in China, Review on Applied Economics, vol.01, pp. 78-82, 2011.

[2]Tian Xin,Sui Yang, Comprehensive Evaluation of Bank's Performance Using TOPSIS Model Based on Entropy Weights, Mathematical Theory and Applications, vol.33,pp: 96-102,2013.

[3]Meng Qingsheng, Information theory,in Xi'an Jiao Tong University press,Xian,China,1989.

[4]Zhang Qishan,Guo Xijiang,Deng Julong, Grey relational entropy analysis method, SYSTEMS ENGINEERING ---THEORY \& PRACTICE, vol.08,pp: 07-11,1996.

[5]Mi Chuanmin, Liu Sifeng,Dang Yaoguo,Fang Zhigeng, Research on grey entropy weight clustering decision method, Systems Engineering and Electronics, vol.28,pp: 1823-1825,2006.

[6]Liu Li,Zhou Jianzhong, Yang Li,Zhang Yongchuan, Application of grey clustering based on entropy weight in flood disaster assessment, Journal of Natural Disasters, vol.19,pp: 214-218,2010.

[7]Wang Jiheng, Yan Xiaoting, Analysis on the performance evaluation of listed commercial banks in the first half of 2013, China Economic \& Trade Herald,vol.04,pp:37-38,2014.

[8]Bi Hezheng,Lu Jian,Zhu Shengxue, A highway intersection safety evaluation and grey clustering based on combination weighting, Journal of Wuhan University of Technology, vol.37,pp:54-60,2015.

[9]Qian Lili,Liu Sifeng,Xie Naiming. Grey clustering model based on the information of entropy weight and interval grey number, Systems Engineering and Electronics, vol.38,pp: $352-356,2016$.

[10]Liu Sifeng,Dang Yaoguo,Fang Zhigeng, Grey system theory and its application,in Science Press, Beijing,China, 2004. 